\title{
Distribution of Random Correlation Matrices: Hyperspherical Parameterization of the Cholesky Factor
}

\author{
Mohsen Pourahmadi ${ }^{\mathrm{a}, *}$, Xiao Wang ${ }^{\mathrm{a}}$ \\ ${ }^{a}$ Department of Statistics, Texas A\&M University, College Station, TX, U.S.A.
}

\begin{abstract}
We study the distribution of random correlation matrices using the hyperspherical parameterization of their Cholesky factors and the distributions of the related angles. We highlight the roles of this procedure in generating high-dimensional correlation matrices.
\end{abstract}

Keywords: Jacobian, Simulation, Positive-definiteness, Reparameterization

\section{INTRODUCTION}

The correlation matrix is a key tool in multivariate statistics and is widely used in many areas of applications. In particular, generating random or "typical" correlation matrices plays increasingly important roles in testing various statistical methodologies and algorithms such as subset selection in multiple regression [1], sampling methods [2], signal processing [3] and finance [4]. For clarity, in what follows we restrict our attention to positive-definite correlation matrices.

A valid $n \times n$ correlation matrix $R=\left(r_{i, j}\right)$ is symmetric and positive-definite with 1 's on the diagonal. Observing the latter constraint when simulating and modeling large correlation matrices makes these tasks more challenging than their counterparts for covariance matrices. Most of the existing algorithms for generating random correlation matrices [3] yield the distribution of certain features like their entries [5] eigenvalues, condition numbers and norms, but not the distribution of the whole correlation matrix. They are usually based on random eigenvalues and random orthogonal matrices or random Gram matrices of the form $T T^{\prime}$ where $T$ is an $n \times m \quad(m \geq n)$ matrix whose rows are vectors of unit length selected randomly from the unit $m$-sphere. Deriving the full distribution of a random correlation matrix constructed from these procedures has remained elusive. Exceptions are an important but largely ignored classic result in Muirhead [6, p.148] which provides the full distribution of the sample correlation matrix of sample data generated from multivariate spherically symmetric distributions, and a recent method due to Joe [7] and Lewandowski et al. [8] based on unconstrained reparameterization of $R$ using its sequential partial correlations which vary freely in the range

*Correspondence to: 3143 TAMU, College Station, TX 77843-3143, U.S.A. Wang)

Email addresses: pourahm@stat.tamu.edu (Mohsen Pourahmadi), xwang@stat.tamu.edu (Xiao

URL: http://www.stat.tamu.edu/ pourahm (Mohsen Pourahmadi) 
$(-1,1)$. By specifying independent univariate extended beta distributions on $(-1,1)$ for the $n(n-1) / 2$ partial correlations, Joe [7] and Lewandowski et al. [8] show that the distribution of the resulting random correlation matrix is proportional to a power of its determinant and hence invariant to the order used in computing the sequence of partial correlations. It hinges on two important formulas expressing the determinants of $R$ and the Jacobian of the transformation from $R$ to the sequential partial correlations.

In the last two decades there has been great interests in unconstrained reparameterization of correlation matrices using the hyperspherical coordinates. The more successful methods rely on the Cholesky decomposition $R=B B^{\prime}$ of a correlation matrix, $[9,10,11]$, and the hyperspherical parameterization of its lower triangular Cholesky factor $B$ using the $n(n-1) / 2$ unique angles $\theta_{i, j}$ which vary freely in the range $(0, \pi)$. It has the nice feature of swapping a constrained correlation matrix $R$ with a matrix of angles $\Theta=\left(\theta_{i, j}\right)$ whose nonredundant entries are not (functionally) related to each other. The full potential of this important reparameterization in finding the distribution of $R$ has not been explored fully. However, we note that Numpacharoen and Atsawarungruangkit [12] uses it in generating random correlation matrices with no guarantee on positive-definiteness, and Mittelbach et al. [13] exploits it in sampling uniformly from the set of positive-definite matrices with a trace constraint.

In this paper, motivated by the distinguished role of the Cholesky decomposition in modeling covariance matrices [14, 15], we propose a direct and succinct method for finding the distribution and generating random correlation matrices. It avoids the need to go through the concepts of partial correlations and the theory of vines as in Lewandowski et al. [8]. However, as in Joe [7] and Lewandowski et al. [8], expressing the determinant of $R$ and the Jacobian of the transformation from the angles to the correlations plays a central role in selecting the distribution of the angles so that the distribution of the correlation matrix is proportional to a power of its determinant, and thus invariant to the choice of coordinates in the Cholesky decomposition. In particular, we specify special distributions for the angles so that the correlation matrix has a uniform density on the convex set $M_{n}$ of all positive-definite correlation matrices.

The outline of the paper is as follows: The reparameterization of the Cholesky factor, finding the $\operatorname{det}(R)$ and the Jacobian of the transformation leading to the distribution of $R$ are presented in Sections 2. In Section 3, we present an algorithm for generating random correlation matrices and compare the performance of our method with earlier methods. The computational complexity of our method for generating random correlation matrices is $O\left(n^{3}\right)$ compared to $O\left(n^{4}\right)$ of the Joe [7] proposal based on partial correlations, and $O\left(n^{3}\right)$ of the Lewandowski et al. [8] method using the partial correlations defined on C-vines, respectively.

\section{Distribution of Random Correlation Matrices}

The idea of reparameterizing the Cholesky factor of a covariance matrix using the hyperspherical coordinates is due to Pinheiro and Bates [9, Section 2.3]. For correlation matrices, an early and naive version was proposed by Rebonato and Jäckel [10], however, Rapisarda et al. [11] develops a more complete setup with the full geometrical implications of the idea. We summarize some preliminary and relevant results in the following: 
Lemma 1. (a) A positive-definite correlation matrix $R=\left(r_{i, j}\right)$ can be factorized as $R=$ $B B^{\prime}$ where $B=\left(b_{i j}\right)$ is a lower triangular matrix with $b_{1,1}=1, b_{i, 1}=\cos \theta_{i, 1}, i=2, \ldots, n$ and

$$
b_{i, j}=\left\{\begin{aligned}
\prod_{k=1}^{j-1} \sin \theta_{i, k} & \text { for } i=j, \\
\cos \theta_{i, j} \cdot \prod_{k=1}^{j-1} \sin \theta_{i, k} & \text { for } 2 \leq j \leq i-1 .
\end{aligned}\right.
$$

or in matrix form

$$
B=\left[\begin{array}{ccccc}
1 & 0 & 0 & \cdots & 0 \\
\cos \theta_{2,1} & \sin \theta_{2,1} & 0 & \cdots & 0 \\
\cos \theta_{3,1} & \cos \theta_{3,2} \sin \theta_{3,1} & \sin \theta_{3,2} \sin \theta_{3,1} & \cdots & 0 \\
\cos \theta_{4,1} & \cos \theta_{4,2} \sin \theta_{4,1} & \cos \theta_{4,3} \sin \theta_{4,2} \sin \theta_{4,1} & \cdots & 0 \\
\vdots & \vdots & \vdots & \cdots & \vdots \\
\cos \theta_{n, 1} & \cos \theta_{n, 2} \sin \theta_{n, 1} & \cos \theta_{n, 3} \sin \theta_{n, 2} \sin \theta_{n, 1} & \cdots & \prod_{k=1}^{n-1} \sin \theta_{n, k}
\end{array}\right],
$$

where $\theta_{i, j}, i>j$, are some angles.

(b) The matrix $B$ is unique if its diagonal entries are positive or equivalently if the angles are restricted to the range $(0, \pi)$. Furthermore, with $c_{i, j}=\cos \theta_{i, j}$ and $s_{i, j}=\sin \theta_{i, j}$, we have

$$
r_{i, j}=c_{i, 1} c_{j, 1}+\sum_{k=2}^{i-1} c_{i, k} c_{j, k} \prod_{l=1}^{k-1} s_{i, l} s_{j, l}+c_{j, i} \prod_{l=1}^{i-1} s_{i, l} s_{j, l}, \quad 1 \leq i<j \leq n,
$$

and the transformation from $R$ to $\Theta=\left(\theta_{i, j}\right)$ is one-to-one, where $\theta_{i, j}=0$ for $i \leq j$. (c) $\operatorname{det}(R)=\prod_{j=1}^{n-1} \prod_{i=j+1}^{n} \sin ^{2} \theta_{i, j}$.

Proof of the lemma is relegated to the Appendix. The relations (1)-(2) provide a one-toone mapping from a general correlation matrix $\mathrm{R}$ to the angles in $\Theta$ when the diagonal entries of the Cholesky factor are required to be positive.

The presence of products of $\sin \theta_{i, j}, i>j$, on the diagonal of $B$ guarantees that its diagonal entries are positive if the range of the angles is restricted to $(0, \pi)$, instead of the customary range $[0,2 \pi)$, see Pinheiro and Bates [9] and Rapisarda et al. [11]. In general, the total number of parameters in $\Theta$ is $\frac{n(n-1)}{2}$, which is the same as the number of free parameters in an unstructured correlation matrix. Furthermore, the angles $\theta_{i, j}$ 's can be expressed in term of the entries of $R$ through those of $B$ using the following:

$$
\theta_{i, 1}=\arccos \left(b_{i, 1}\right), i=2, \ldots, n, \theta_{i, j}=\arccos \left(b_{i, j} / \prod_{k=1}^{j-1} \sin \left(\arccos \left(b_{i, k}\right)\right)\right), 2 \leq j<i \leq n .
$$

The forms of the Jacobian of the transformation $R \rightarrow \Theta$, and $\operatorname{det}(R)$ provide useful clues on choosing suitable densities for the angles so that the distribution of $R$ has simple forms with desirable properties. More precisely, for $\theta_{i, j}$ 's independent univariate random variables with densities $g_{i, j}(\cdot)$, the joint density of the correlation matrix $R=\left(r_{i, j}\right)$ is given by

$$
f(R)=\prod_{i=2}^{n} \prod_{j=1}^{i-1} g_{i, j}\left(\theta_{i, j}\right)|J|,
$$


where $|J|$ is the determinant of the Jacobian of $\left(r_{i, j}\right)$ with respect to $\left(\theta_{i, j}\right)$, found next.

Lemma 2. Consider arranging all the nonredundant elements of the matrices $\Theta$ and $R$ into the vectors $\Theta=\left(\theta_{2,1}, \theta_{3,1}, \theta_{3,2}, \theta_{4,1}, \theta_{4,2}, \theta_{4,3}, \cdots, \theta_{n, 1}, \theta_{n, 2}, \theta_{n, 3}, \cdots, \theta_{n, n-1}\right)$ and $R=$ $\left(r_{2,1}, r_{3,1}, r_{3,2}, r_{4,1}, r_{4,2}, r_{4,3}, \cdots, r_{n, 1}, r_{n, 2}, r_{n, 3}, \cdots, r_{n, n-1}\right)$, respectively. Then (i) the Jacobian $\left(\frac{\partial R}{\partial \Theta}\right)$ is a lower triangular matrix and (ii) its determinant is given by

$$
\operatorname{det}\left(\frac{\partial R}{\partial \Theta}\right)=\prod_{i=2}^{n} \prod_{j=1}^{i-1} \frac{\partial r_{i, j}}{\partial \theta_{i, j}}=(-1)^{\sum_{h=0}^{n-1} h} \times \prod_{j=1}^{n-1}\left(\prod_{i=j+1}^{n} s_{i, j}\right)^{n-j}
$$

The proof is given in the Appendix. Next, we choose the distributions of the angles $\theta_{i, j}^{\prime} \mathrm{s}$ in such a way that the distribution of $R$ is proportional to a power of its determinant.

Theorem 1. With matrices $R, B, \Theta$ as before and for a fixed $j=1,2, \ldots n-1$, let the random variables in the $j$ th column of $\Theta$ be independent and identically distributed as

$$
\theta_{i, j} \sim g_{j}(\theta) \propto(\sin \theta)^{2 k+n-j} I(0<\theta<\pi), i=j+1, j+2, \ldots, n,
$$

where $k \geq 0$ is a constant. Then,

(a) the joint distribution of the correlations

$$
R=\left(r_{2,1}, r_{3,1}, r_{3,2}, r_{4,1}, r_{4,2}, r_{4,3}, \cdots, r_{n, 1}, r_{n, 2}, r_{n, 3}, \cdots, r_{n, n-1}\right)
$$

is given by

where

$$
f(R)=c_{n}(k)\left(\prod_{j=1}^{n} \prod_{\ell=1}^{j-1} \sin ^{2} \theta_{j, \ell}\right)^{k}=c_{n}(k)[\operatorname{det}(R)]^{k},
$$

is the normalizing constant.

$$
c_{n}(k)=\prod_{j=1}^{n-1}\left[\frac{\Gamma\left(\frac{2 k+j}{2}+1\right)}{\sqrt{\pi} \Gamma\left(\frac{2 k+j+1}{2}\right)}\right]^{j},
$$

(b) for $k=0$, the distribution in (a) is a uniform distribution on $M_{n}$, the set of all $n \times n$ positive-definite correlation matrices. The volume of $M_{n}$ is given by

$$
1 / c_{n}(0)=\prod_{i=1}^{n-1}\left(\int_{0}^{\pi} \sin ^{i} \theta d \theta\right)^{i}=\prod_{i=1}^{n-1} B\left(\frac{i+1}{2}, \frac{1}{2}\right)^{i},
$$

where $B(\cdot, \cdot)$ is the Beta function, and matches the following two alternative formulas

$$
\frac{\pi^{\frac{n(n-1)}{4}} \prod_{i=1}^{n} \Gamma\left(\frac{n+1}{2}-\frac{i-1}{2}\right)}{\Gamma\left(\frac{n+1}{2}\right)^{n}}, \quad \prod_{i=1}^{n} 2^{(i-1)^{2}} B\left(\frac{1}{2} i, \frac{1}{2} i\right)^{i-1}
$$

given, respectively, by Wong et al. [15, Lemma A.2] and Joe [7, p. 183].

(c) The marginal density of each element $r_{i, j}, j=1,2, \cdots n-1, i=j+1, j+2, \cdots, n$ of the correlation matrix $R$ is proportional to $\left(1-r^{2}\right)^{k+\frac{n}{2}-1}$, i.e., the $\operatorname{Beta}\left(k+\frac{n}{2}, k+\frac{n}{2}\right)$ distribution on $(-1,1)$. 
Among other applications, the result in (b) is useful for computing the probability that a random symmetric matrix with 1's on the diagonal and other entries drawn from i.i.d. $U(-1,1)$ is positive-definite. In fact, the probability is

$$
\frac{\operatorname{Volume}\left(M_{n}\right)}{\operatorname{Volume}\left((-1,1)^{\frac{n(n-1)}{2}}\right)}=\frac{\prod_{j=1}^{n-1}\left[\frac{\sqrt{\pi} \Gamma\left(\frac{j+1}{2}\right)}{\Gamma\left(\frac{j}{2}+1\right)}\right]^{j}}{2^{\frac{n(n-1)}{2}}}=\prod_{j=1}^{n-1}\left[\frac{\sqrt{\pi} \Gamma\left(\frac{j+1}{2}\right)}{2 \Gamma\left(\frac{j}{2}+1\right)}\right]^{j}<\prod_{j=1}^{n-1}\left[\frac{\sqrt{\pi}}{2}\right]^{j}=\left[\frac{\sqrt{\pi}}{2}\right]^{\frac{n(n-1)}{2}},
$$

which converges to 0 for $n$ large, see Böhm and Hornik [16].

\section{Generating Random Correlation Matrices}

In this section, using the hyperspherical parameterization (HP) of the Cholesky factor we present a simple procedure for generating random correlation matrices with guaranteed positive-definiteness and distribution given in Theorem 1 (a). Our HP algorithm is based on the following two steps:

Step 1: For a fixed $k \geq 0$, use the inverse transformation sampling [17, Section 2.2] to draw $\theta_{i, j}$ 's according to the distribution in (5).

Step 2: Compute the lower triangular matrix $B$ in (1) using $\theta_{i, j}$ and construct the correlation matrix $R=B B^{\prime}$.

We conduct a simulation study to compare the computational performance of the HP algorithm with the following five methods:

1. Reject Sampling(RS) constructs a random matrix $R$ by generating $r_{i, j} \sim U(-1,1), i>$ $j$, independently. The matrix will be rejected as a correlation matrix if it is not positivedefinite.

2. The NA method, due to Numpacharoen and Atsawarungruangkit [12], also relies on the hyperspherical parameterization of the Cholesky factor to generate random correlation matrices. It finds theoretical boundaries for each correlation coefficient and requires choosing a threshold level $K$ for the difference between the upper and lower bounds. The bounds are found by noting that the $\theta_{i, j}$ 's take values in $(0, \pi), \sin \theta_{i, j}$ is positive and the cosine takes values in $(-1,1)$. Then, using $(2)$ the lower and upper bounds of each correlation coefficient is found by setting $\cos \theta_{i, j}$ to -1 or 1 . The threshold level $K$ is a key parameter which controls the stability of the method, with larger values leading to more stable methods, the suggested value is $K=0.01$. For a chosen threshold level, the validity of a generated correlation matrix must be checked by computing its minimum eigenvalue which can be computationally expensive for large $n$.

3. Randcorr function (RC) [18] in MATLAB is based on a popular algorithm in Bendel and Mickey [2] which takes a matrix having specified eigenvalues and uses a finite sequence of Givens rotations to introduce 1's on the diagonal. The RC gives improved formulae for computing the rotations and the resulting algorithm is known to be numerically stable.

4. Partial Correlation (PC) [7] and C-Vine in [8] relies on the sequential partial correlations and $\mathrm{C}-\mathrm{Vine}$ structure to reparameterize and generate a correlation matrix. 
5. Sample Correlation (SC) [6, p.148] computes the sample correlation matrix of random samples generated from multivariate spherically symmetric distributions.

The performance of each algorithm is measured by the expected run time $T_{\exp }$ calculated from the average run time $T_{\text {avg }}$ divided by $P_{\text {valid }}$, the probability of a generated correlation matrix being valid. Note that for the first two methods $T_{\text {avg }}$ includes the time taken to construct the correlation matrix as well as checking its positive-definiteness. The number of replications in the simulation is 1000 .

$$
\text { comparing }
$$

The simulation results shown in the Table 1 reveal the numerical instability of the RS and NA algorithms in [12]. Using the RS method it is almost impossible to genqrate a valid random correlation matrix of dimension greater than 7 , see Böhm and Hornik [16]. The NA method is unstable for larger dimensions $(n=300,400,500)$ which might be due to the choice of the threshold parameter $K$. The need to choose $K$ reduces the practical appeal of the NA method in generating large random correlation matrices. The theoretical results and the simulation experiments show that the RC [18], the C-Vine [8], the HP and the SC methods are stable in the sense that random correlation $p$ atrices generated by these four methods are guaranteed to be positive definite. From the HP and $\mathrm{C}$-Vine columns, it is evident that the $\mathrm{HP}$ algorithm is faster for larger $\mathrm{n}$ and is slower for smaller $n$. All programs have been run on a desktop computer with Intel Core $(4 \times 3.40 \mathrm{GHz})$ processor, 7.6 GB of RAM memory and Kernel Linux x86_64 operating system.

\section{Discussion and Future Research}

We have shown that reparameterizing the Cholesky factor of a correlation matrix using hyperspherical coordinates provides a simpler and more succinct method for finding the distribution and simulating random correlation matrices compared to four viable and competing existing methods. The unconstrained nature of the reparameterization of the Cholesky factor makes it also suitable for modeling correlation matrices using covariates, see Zhang et al. [19]. One may rely on Bayesian techniques and impose priors on the angles to shrink them to zero or other target values. In the case of sequential partial correlations, in Daniels and Pourahmadi [20] a flexible prior on $R$ is constructed using independent extended Beta priors on the unconstrained partial correlations. The corresponding Bayesian approach based on the hyperspherical reparameterization of a correlation matrix is an open problem. Furthermore, the distribution of the random correlation matrix generated in Madar [21] and its computational complexity need to be investigated.

Acknowledgments: M. Pourahmadi is supported by NSF grant DMS-1309586.

\section{Appendix: Proofs}

Proof of Lemma 1: (a) Existence of a lower triangular matrix $B$ for a positive-definite matrix $R$ is a basic result in linear algebra. Parameterizing the rows of $B$ using the hyperspherical coordinates guarantees that the diagonal entries of $B B^{\prime}$ are equal to one. 
This is obvious for the $(1,1)$ and $(2,2)$ entries, but in general

$$
\begin{aligned}
r_{i, i} & =\sum_{k=1}^{n} b_{i, k} b_{i, k}=b_{i, 1} b_{i, 1}+\sum_{k=2}^{i-1} b_{i, k} b_{i, k}+b_{i, i} b_{i, i} \\
& =c_{i, 1}^{2}+\sum_{k=2}^{i-1}\left(c_{i, k} \prod_{l=1}^{k-1} s_{i, l}\right)^{2}+\left(\prod_{l=1}^{i-1} s_{i, l}\right)^{2}=c_{i, 1}^{2}+\sum_{k=2}^{i-2}\left(c_{i, k} \prod_{l=1}^{k-1} s_{i, l}\right)^{2}+\left(\prod_{l=1}^{i-2} s_{i, l}\right)^{2} \\
& =c_{i, 1}^{2}+\sum_{k=2}^{i-3}\left(c_{i, k} \prod_{l=1}^{k-1} s_{i, l}\right)^{2}+\left(\prod_{l=1}^{i-3} s_{i, l}\right)^{2}=\cdots=c_{i, 1}^{2}+\left(c_{i, 2} s_{i, 1}\right)^{2}+\left(s_{i, 2} s_{i, 1}\right)^{2}=c_{i, 1}^{2}+s_{i, 1}^{2}=1
\end{aligned}
$$

$$
\begin{aligned}
r_{i, j} & =\sum_{k=1}^{n} b_{i, k} b_{j, k}=b_{i, 1} b_{j, 1}+\sum_{k=2}^{i-1} b_{i, k} b_{j, k}+b_{i, i} b_{j, i} \\
& =c_{i, 1} c_{j, 1}+\sum_{k=2}^{i-1}\left(c_{i, k} \prod_{l=1}^{k-1} s_{i, l}\right)\left(c_{j, k} \prod_{l=1}^{k-1} s_{j, l}\right)+\prod_{l=1}^{i-1} s_{i, l}\left(c_{j, i} \prod_{l=1}^{i-1} s_{j, l}\right) \\
& =c_{i, 1} c_{j, 1}+\sum_{k=2}^{i-1} c_{i, k} c_{j, k} \prod_{l=1}^{k-1} s_{i, l} s_{j, l}+c_{j, i} \prod_{l=1}^{i-1} s_{i, l} s_{j, l},
\end{aligned}
$$

for $1 \leq i<j \leq n$.

(c) $\operatorname{det}(R)=\operatorname{det}\left(B B^{\prime}\right)=[\operatorname{det}(B)]^{2}=\prod_{j=1}^{n-1} \prod_{i=j+1}^{n} \sin ^{2} \theta_{i, j}$.

Proof of Lemma 2: (i) An element in the upper-diagonal of the Jacobian is of the form $\frac{\partial r_{i, j}}{\partial \theta_{p, q}}$ with $1 \leq j<i \leq n$ and $p>i$ or $q>j$. It is seen in (2) that $r_{i, j}$ is a function of the angles $\theta_{s, t}$ 's with smaller row and column indices, i.e., $s<i$ and $t<j$, thus $\frac{\partial r_{i, j}}{\partial \theta_{p, q}}=0$ and the Jacobian is a lower triangular matrix.

(ii) One can prove (4) using brute force by computing the diagonal entries $\frac{\partial r_{i, j}}{\partial \theta_{i, j}}$ of the Jacobian matrix. However, we prefer the following simpler induction method. Assume (4) holds for $\mathrm{n}$, we prove it for $\mathrm{n}+1$. Note that for $1 \leq j<i \leq n+1$,

and

$$
\frac{\partial r_{i, j}}{\partial \theta_{i, j}}= \begin{cases}-s_{i, 1} & \text { if } j=1 \\ -s_{i, j} \prod_{l=1}^{j-1} s_{j, l} s_{i, l} & \text { if } j \neq 1\end{cases}
$$

$\prod_{i=1}^{n} \frac{\partial r_{n+1, j}}{\partial \theta_{n+1, j}}=\left(-s_{n+1,1}\right) \times \prod_{k=2}^{n}\left(-s_{n+1, k}\right) \prod_{l=1}^{k-1} s_{k, l} s_{n+1, l}=(-1)^{n} \times s_{n+1, n} \prod_{j=1}^{n-1}\left(s_{n+1, j}\right)^{n+1-j} \prod_{i=j+1}^{n} s_{i, j}$. 
Using (4) which holds for n by the induction's assumption, we have,

$$
\begin{aligned}
\operatorname{det}\left(\frac{\partial R}{\partial \Theta}\right) & =\prod_{i=2}^{n+1}\left\{\prod_{j=1}^{i-1} \frac{\partial r_{i, j}}{\partial \theta_{i, j}}\right\}=\prod_{i=2}^{n}\left\{\prod_{j=1}^{i-1} \frac{\partial r_{i, j}}{\partial \theta_{i, j}}\right\} \times \prod_{j=1}^{n} \frac{\partial r_{n+1, j}}{\partial \theta_{n+1, j}} \\
& =(-1)^{\sum_{h=1}^{n-1} h} \times \prod_{j=1}^{n-1}\left(\prod_{i=j+1}^{n} s_{i, j}\right)^{n-j} \times(-1)^{n} \times s_{n+1, n} \prod_{j=1}^{n-1}\left(s_{n+1, j}\right)^{n+1-j} \prod_{i=j+1}^{n} s_{i, j} \\
& =(-1)^{\sum_{h=1}^{n} h} \times \prod_{j=1}^{n}\left(\prod_{i=j+1}^{n+1} s_{i, j}\right)^{n+1-j}
\end{aligned}
$$

Proof of Theorem 1 (a) Let $k_{j}$ be the normalizing constant for the distribution of $\theta_{i, j}$. Then, the joint distribution of $R$ is:

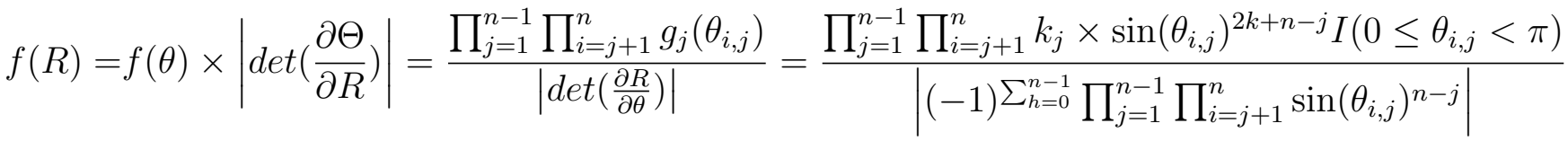

$$
\begin{aligned}
& =\prod_{j=1}^{n-1} \prod_{i=j+1}^{n} k_{j} I\left(0 \leq \theta_{i, j}<\pi\right) \times\left[\prod_{j=1}^{n-1} \prod_{i=j+1}^{n} \sin ^{2} \theta_{i, j}\right]^{k}=c_{n}(k) \times \operatorname{det}^{k}(R) \propto \operatorname{det}^{k}(R),
\end{aligned}
$$

where

$$
\begin{aligned}
c_{n}(k) & =\frac{1}{\prod_{j=1}^{n-1}\left(\int_{0}^{\pi} \sin ^{2 k+j}(\theta) d \theta\right)^{j}}=\frac{1}{\prod_{j=1}^{n-1} B^{j}\left(\frac{1}{2}(2 k+j+1), \frac{1}{2}\right)} \\
& =\prod_{j=1}^{n-1} \frac{\Gamma^{j}\left(\frac{2 k+j}{2}+1\right)}{\Gamma^{j}\left(\frac{2 k+j+1}{2}\right) \Gamma^{j}\left(\frac{1}{2}\right)}=\prod_{j=1}^{n-1}\left[\frac{\Gamma\left(\frac{2 k+j}{2}+1\right)}{\sqrt{\pi} \Gamma\left(\frac{2 k+j+1}{2}\right)}\right]^{j} .
\end{aligned}
$$

(b) From (a), it is obvious that when $k=0$, the joint distribution is a uniform distribution on $M_{n}$ and hence its volume

$$
V_{n}=\frac{1}{c_{n}(0)}=\prod_{j=1}^{n-1}\left[\frac{\sqrt{\pi} \Gamma\left(\frac{j+1}{2}\right)}{\Gamma\left(\frac{j}{2}+1\right)}\right]^{j}
$$

It is trivial that (i) (ii) hold for $n=1$. Assuming they hold for $n$, we'll show they hold 
for $n+1$ :

$$
\begin{aligned}
& \text { (i) } \prod_{i=1}^{n}\left[\frac{\sqrt{\pi} \Gamma\left(\frac{i+1}{2}\right)}{\Gamma\left(\frac{i}{2}+1\right)}\right]^{i}=\left[\frac{\sqrt{\pi} \Gamma\left(\frac{n+1}{2}\right)}{\Gamma\left(\frac{n}{2}+1\right)}\right]^{n} \prod_{i=1}^{n-1}\left[\frac{\sqrt{\pi} \Gamma\left(\frac{i+1}{2}\right)}{\Gamma\left(\frac{i}{2}+1\right)}\right]^{i} \\
&=\left[\frac{\sqrt{\pi} \Gamma\left(\frac{n+1}{2}\right)}{\Gamma\left(\frac{n}{2}+1\right)}\right]^{n} \frac{\pi^{\frac{n(n-1)}{4}} \prod_{i=1}^{n} \Gamma\left(\frac{n+1}{2}-\frac{i-1}{2}\right)}{\Gamma\left(\frac{n+1}{2}\right)^{n}}=\frac{\pi^{\frac{n(n+1)}{4}} \prod_{i=1}^{n+1} \Gamma\left(\frac{n+2}{2}-\frac{i-1}{2}\right)}{\Gamma\left(\frac{n+2}{2}\right)^{n+1}} . \\
& \text { (ii) } \sqrt{\pi} \times i ! \stackrel{\star}{=} 2^{i} \Gamma\left(\frac{i+1}{2}\right) \Gamma\left(\frac{i+2}{2}\right) \Rightarrow \frac{\sqrt{\pi} \Gamma\left(\frac{i+1}{2}\right)}{\Gamma\left(\frac{i}{2}+1\right)}=2^{i} \frac{\Gamma\left(\frac{i+1}{2}\right) \Gamma\left(\frac{i+1}{2}\right)}{\Gamma(i+1)} \\
& \Rightarrow \prod_{i=1}^{n}\left[\frac{\sqrt{\pi} \Gamma\left(\frac{i+1}{2}\right)}{\Gamma\left(\frac{i}{2}+1\right)}\right]^{i}=\left[\frac{\sqrt{\pi} \times \Gamma\left(\frac{n+1}{2}\right)}{\Gamma\left(\frac{n}{2}+1\right)}\right]^{n} \times \prod_{i=1}^{n-1}\left[\frac{\sqrt{\pi} \Gamma\left(\frac{i+1}{2}\right)}{\Gamma\left(\frac{i}{2}+1\right)}\right]^{i} \\
&=2^{n^{2}}\left[\frac{\Gamma\left(\frac{n+1}{2}\right) \Gamma\left(\frac{n+1}{2}\right)}{\Gamma(n+1)}\right]^{n} \times \prod_{i=1}^{n} 2^{(i-1)^{2}} B\left(\frac{1}{2} i, \frac{1}{2} i\right)^{i-1}=\prod_{i=1}^{n+1} 2^{(i-1)^{2}} B\left(\frac{1}{2} i, \frac{1}{2} i\right)^{i-1} .
\end{aligned}
$$

Now, it only remains to show the validity of the equation $\star$. It is obviously correct for $i=1$. Using induction again, assuming that it holds for $i$, we obtain

$\sqrt{\pi} \cdot(i+1) !=\sqrt{\pi} \cdot i ! \cdot(i+1)=2^{i+1} \cdot \Gamma\left(\frac{i+2}{2}\right) \cdot \Gamma\left(\frac{i+1}{2}\right) \cdot \frac{i+1}{2}=2^{i+1} \cdot \Gamma\left(\frac{i+2}{2}\right) \cdot \Gamma\left(\frac{i+3}{2}\right)$.

(c) Since permuting the rows and columns of $R$ does not alter its distribution, it suffices to show that $r_{n, 1}$ has the distribution $\operatorname{Beta}\left(k+\frac{n}{2}, k+\frac{n}{2}\right)$ on the interval $(-1,1)$. From $\theta_{n, 1} \propto \sin (\theta)^{2 k+n-1} I(0<\theta<\pi)$, it follows that,

$$
r_{n, 1}=\cos \left(\theta_{n, 1}\right) \propto[\sin (\arccos (r))]^{2 k+n-1} \times \frac{d \arccos (r)}{d r} \propto\left(1-r^{2}\right)^{k+\frac{n}{2}-1} .
$$

\section{References}

[1] D. G. Johnson and W. J. Welch. The generation of pseudo- random correlation matrices. Journal of Statistical Computation and Simulation, 11:55-69, 1980.

[2] R. B. Bendel and M. R. Mickey. Population correlation matrices for sampling experiments. Communications in Statistics - Simulation and Computation, 7:163-182, 1978.

[3] R. B. Holmes. On random correlation matrices. SIAM J. Matrix Anal. Appl., 12: 239-272, 1991.

[4] R. Rebonato and P. Jäckel. The most general methodology for creating a valid correlation matrix for risk management and option pricing purposes. Journal of Risk, 01:279-282, 2000.

[5] G. Marsaglia and I. Olkin. Generating correlation matrices. j-SIAM-J-SCI-STATCOMP, 5:470-475, 1984.

[6] R. J. Muirhead. Aspects of Multivariate Statistical Theory. Wiley-Interscience, Ann Arbor, MI., 1982. 
[7] H. Joe. Generating random correlation matrices based on partial correlations. J. of Multivariate Analysis, 97:2177-2189, 2006.

[8] D. Lewandowski, D. Kurowicka, and H. Joe. Generating random correlation matrices based on vines and extended onion method. Journal of Multivariate Analysis, 100 (9):1989 - 2001, 2009.

[9] J.D. Pinheiro and D.M. Bates. Unconstrained parameterizations for variancecovariance matrices. Stat. Comp., 6:289-366, 1996.

[10] R. Rebonato and P. Jäckel. The most general methodology to create a valid correlation matrix for risk management and option pricing purposes. J. of Risk, 2:17-27, 2000 .

[11] F. Rapisarda, D. Brigo, and F. Mercurio. Parameterizing correlations: a geometric interpretation. IMA Journal of Management Mathematics, 18:55-73, 2007.

[12] K. Numpacharoen and A. Atsawarungruangkit. Generating correlation matrices based on the boundaries of their coefficients. PloS one, 7, 2012.

[13] M. Mittelbach, B. Matthiesen, and E. A. Jorswieck. Sampling uniformly from the set of positive definite matrices with trace constraint. IEEE Transactions on Signal Processing, 60(5):2167-2179, May 2012.

[14] M. Pourahmadi. Joint mean-covariance models with applications to longitudinal data: Unconstrained parameterisation. Biometrika, 86:677-690, 1999.

[15] F. Wong, C.K. Carter, and R. Kohn. Efficient estimation of covariance selection models. Biometrika, 90:809-830, 2003.

[16] W. Böhm and K. Hornik. Generating random correlation matrices by the simple rejection method: Why it does not work. Statistics and Probability Letters, 87(C): $27-30,2014$.

[17] Luc Devroye. Non-Uniform Random Variate Generation. Springer-Verlag, New York, 1986.

[18] P. I. Davies and N. J. Higham. Numerically stable generation of correlation matrices and their factors. BIT, 40:640-651, 2000.

[19] W. Zhang, C. Leng, and C. Y. Tang. A joint modeling approach for longitudinal studies. Journal of the Royal Statistical Society Series B, 77:219-238, 2015.

[20] M.J. Daniels and M. Pourahmadi. Modeling covariance matrices via partial autocorrelations. Journal of Multivariate Analysis, 100:2352-2363, 2008.

[21] V. Madar. Direct formulation to cholesky decomposition of a general nonsingular correlation matrix. to appear in Statistics and Probability Letters, available at http://arxiv.org/abs/1412.1181, 2015. 


\begin{tabular}{|l|ll|llllll|}
\hline & $P_{\text {valid }}$ & & \multicolumn{7}{l|}{$T_{\text {exp }}$} \\
\hline $\mathrm{n}$ & RS & NA & RS & NA & RC & C-VINE & HP & SC \\
\hline 3 & 100 & 100 & $6.70 \mathrm{E}-04$ & $1.30 \mathrm{E}-04$ & $2.90 \mathrm{E}-04$ & $5.00 \mathrm{E}-05$ & $6.00 \mathrm{E}-04$ & $4.50 \mathrm{E}-04$ \\
5 & 100 & 100 & $4.40 \mathrm{E}-03$ & $2.70 \mathrm{E}-04$ & $4.40 \mathrm{E}-04$ & $4.00 \mathrm{E}-04$ & $1.96 \mathrm{E}-03$ & $1.70 \mathrm{E}-03$ \\
7 & 100 & 100 & $3.83 \mathrm{E}-01$ & $5.20 \mathrm{E}-04$ & $5.40 \mathrm{E}-04$ & $6.00 \mathrm{E}-04$ & $3.51 \mathrm{E}-03$ & $4.43 \mathrm{E}-03$ \\
8 & 0 & 100 & $\infty$ & $5.00 \mathrm{E}-04$ & $6.00 \mathrm{E}-04$ & $8.00 \mathrm{E}-04$ & $4.20 \mathrm{E}-03$ & $6.00 \mathrm{E}-03$ \\
10 & 0 & 100 & $\infty$ & $1.20 \mathrm{E}-03$ & $8.00 \mathrm{E}-04$ & $1.50 \mathrm{E}-03$ & $6.91 \mathrm{E}-03$ & $8.81 \mathrm{E}-03$ \\
20 & 0 & 100 & $\infty$ & $5.20 \mathrm{E}-03$ & $2.70 \mathrm{E}-03$ & $9.05 \mathrm{E}-03$ & $2.86 \mathrm{E}-02$ & $3.13 \mathrm{E}-02$ \\
30 & 0 & 100 & $\infty$ & $1.20 \mathrm{E}-02$ & $6.10 \mathrm{E}-03$ & $2.93 \mathrm{E}-02$ & $6.76 \mathrm{E}-02$ & $1.16 \mathrm{E}-01$ \\
40 & 0 & 100 & $\infty$ & $2.17 \mathrm{E}-02$ & $1.29 \mathrm{E}-02$ & $6.92 \mathrm{E}-02$ & $1.28 \mathrm{E}-01$ & $2.21 \mathrm{E}-01$ \\
50 & 0 & 100 & $\infty$ & $3.45 \mathrm{E}-02$ & $2.60 \mathrm{E}-02$ & $1.34 \mathrm{E}-01$ & $2.06 \mathrm{E}-01$ & $4.52 \mathrm{E}-01$ \\
60 & 0 & 100 & $\infty$ & $5.25 \mathrm{E}-02$ & $4.60 \mathrm{E}-02$ & $2.38 \mathrm{E}-01$ & $2.98 \mathrm{E}-01$ & $6.93 \mathrm{E}-01$ \\
70 & 0 & 100 & $\infty$ & $7.07 \mathrm{E}-02$ & $8.37 \mathrm{E}-02$ & $3.66 \mathrm{E}-01$ & $4.14 \mathrm{E}-01$ & $1.09 \mathrm{E}+00$ \\
80 & 0 & 100 & $\infty$ & $9.26 \mathrm{E}-02$ & $1.34 \mathrm{E}-01$ & $5.55 \mathrm{E}-01$ & $5.46 \mathrm{E}-01$ & $1.64 \mathrm{E}+00$ \\
90 & 0 & 100 & $\infty$ & $1.19 \mathrm{E}-01$ & $2.05 \mathrm{E}-01$ & $7.87 \mathrm{E}-01$ & $7.15 \mathrm{E}-01$ & $2.28 \mathrm{E}+00$ \\
100 & 0 & 100 & $\infty$ & $1.49 \mathrm{E}-01$ & $3.15 \mathrm{E}-01$ & $1.08 \mathrm{E}+00$ & $9.01 \mathrm{E}-01$ & $3.08 \mathrm{E}+00$ \\
200 & 0 & 100 & $\infty$ & $6.27 \mathrm{E}-01$ & $4.33 \mathrm{E}+00$ & $8.82 \mathrm{E}+00$ & $3.99 \mathrm{E}+00$ & $2.40 \mathrm{E}+01$ \\
300 & 0 & 90 & $\infty$ & $1.43 \mathrm{E}+00$ & $2.09 \mathrm{E}+01$ & $2.97 \mathrm{E}+01$ & $9.78 \mathrm{E}+00$ & $8.34 \mathrm{E}+01$ \\
400 & 0 & 80 & $\infty$ & $2.54 \mathrm{E}+00$ & $6.49 \mathrm{E}+01$ & $7.16 \mathrm{E}+01$ & $1.82 \mathrm{E}+01$ & $1.91 \mathrm{E}+02$ \\
500 & 0 & 90 & $\infty$ & $4.00 \mathrm{E}+00$ & $1.58 \mathrm{E}+02$ & $1.39 \mathrm{E}+02$ & $2.88 \mathrm{E}+01$ & $3.72 \mathrm{E}+02$ \\
600 & 0 & 100 & $\infty$ & $5.80 \mathrm{E}+00$ & $3.35 \mathrm{E}+02$ & $2.45 \mathrm{E}+02$ & $4.27 \mathrm{E}+01$ & $6.47 \mathrm{E}+02$ \\
\hline
\end{tabular}

Table 1: Percentage of valid random correlation matrices and expected times for the six indicated methods. 